JOURNAL OF

FUNCTION SPACES AND APPLICATIONS

Volume 3, Number 3 (2005), 251-262 (c) 2005, Scientific Horizon

http://www.jfsa.net

\title{
Infinite-dimensional projected dynamics and the 1-dimensional obstacle problem*
}

\author{
Monica-Gabriela Cojocaru
}

(Communicated by Lars-Erik Persson)

2000 Mathematics Subject Classification. Primary 34A36, 37N15; Secondary 58E35, 47H07.

Keywords and phrases. Infinite-dimensional projected dynamical systems and variational inequalities, Sobolev Hilbert space.

\footnotetext{
Abstract. In this paper we present a direct application of the theory of infinite-dimensional projected dynamical systems (PDS) related to the well-known obstacle problem, i.e., the problem of determining the shape of an elastic string stretched over a body (obstacle). While the obstacle problem is static in nature and is solved via variational inequalities theory, we show here that the dynamic problem of describing the vibration movement of the string around the obstacle is solved via the infinite-dimensional theory of projected dynamical systems.
}

\section{Introduction}

The rigorous theory of infinite-dimensional PDS started in 2002, with the works of Isac and Cojocaru (see [14], [4]), and continued in [5], [15]. The most recent theoretical and applicative developments can be found in Cojocaru et al. ([3] and [6]). Such a theory comes as a natural extension to

${ }^{*}$ Research funded by NSERC Discovery Grant No. 045997. 
infinite-dimensional Hilbert spaces of the theory of PDS in finite dimensions which started in the early 90's with the papers of Dupuis and Nagurney [8] and [9].

The finite-dimensional theory has its roots in equilibrium problems and their relation to variational inequality problems (VI). It has applications to economics, operations research, management, to name a few areas, as can be seen in the works by Nagurney et al. (see [18], [19], [20], [21], [22], [25] and the references therein).

The intimate relation between a PDS and an associated VI, in both finite and infinite-dimensional settings, is the catalyst for viewing a PDS as the natural dynamics which "tells the story" of a system around steady states. This relation consists of the fact that any critical point of a PDS coincides with a solution to the associated VI (and viceversa), which solution, in turn, is known to describe a steady state of a physical system.

Exploiting the relation between PDS and VI, we give here an example of how PDS describes the dynamics of an elastic string stretched over a body and subjected to vibrations. The mathematical model uses a discontinuous differential equation, also known as a projected differential equation, defined with respect to a closed, convex subset of a Hilbert Sobolev space. Together with the applications of infinite-dimensional PDS in relation to evolutionary variational inequalities (see [6]), this paper fills an important gap in the applicative potential of infinite-dimensional PDS, which is now under intense investigation. Since a number of boundary value problems can be studied with the help of VI theory (see for example the classical works [2],[16], [24]), the methodology engaged here becomes useful if one is interested in a dynamics of these problems around their steady states.

The paper is organized as follows: Section 2 gives a brief introduction to PDS and their relation to VI problems. Section 3 presents the formulation of the 1-dimensional obstacle problem as a VI, while Section 4 presents the same problem in relation to a projected dynamics. We close with a few concluding remarks and acknowledgments.

\section{Projected dynamical systems and variational inequalities}

We assume the reader is familiar with the concepts of convex cone, polar cone and set-valued mapping. Let $X$ be a Hilbert space and let $K \subset X$ be a non-empty, closed and convex subset. We recall that for each $x \in K$ the set $T_{K}(x)=\overline{\bigcup_{h>0} \frac{1}{h}(K-x)}$ is the tangent cone to $K$ at $x$. The normal cone to the set $K$ at $x$ is the polar cone of $T_{K}(x)$, 
given by $N_{K}(x):=\left\{p \in X \mid\left\langle p, x-x^{\prime}\right\rangle \geq 0, \forall x^{\prime} \in K\right\}$. We also recall that for any $z \in X$, there exists a unique element in $K$, denoted by $P_{K}(z)$, such that $\left\|P_{K}(z)-z\right\|=\inf _{x \in K}\|x-z\|$. This defines a mapping $P_{K}: X \rightarrow K$ given by $z \mapsto P_{K}(z)$, called the projection operator of the space $X$ onto the subset $K$. The properties of the projection operator on Hilbert spaces are well-known (see [25]). Evidently, $P_{K}(x)=x$, for any $x \in K$.

The Gateaux directional derivative of $P_{K}$ is defined as follows (for a proof see [25], Lemma 4.6 or [23], Section 3).

Proposition 2.1. For any $x \in K$ and any element $v \in X$ the limit

$$
\Pi_{K}(x, v):=\lim _{\delta \rightarrow 0^{+}} \frac{P_{K}(x+\delta v)-x}{\delta} \text { exists and } \Pi_{K}(x, v)=P_{T_{K}(x)}(v) .
$$

Let $\Pi_{K}: K \times X \rightarrow X$ be the operator given by $(x, v) \mapsto \Pi_{K}(x, v)$. Note that, for each $x \in K$ and $v \in X$, there exists $n \in N_{K}(x)$ so that $\Pi_{K}(x, v)=v-n$, whenever $v \notin T_{K}(x)$ (see [8], [2], [3]). In other words, $\Pi_{K}$ is discontinuous on the boundary of the set $K$. A detailed treatment of this operator on infinite-dimensional Hilbert spaces can be found in Isac and Cojocaru [15]. Throughout this paper, we shall use the fact that $\Pi_{K}(x, v)=v-n$, whenever $v \notin T_{K}(x)$.

Definition 2.1. Let $X$ be a Hilbert space of arbitrary dimension and $K \subset X$ be a non-empty, closed and convex subset. Let $F: K \rightarrow X$ be a vector field. The differential equation

$$
\frac{d x(t)}{d t}=\Pi_{K}(x(t),-F(x(t)))
$$

is called the projected differential equation ( $\operatorname{PrDE}$ ) associated with $-F$ and $K$.

A PrDE is a special case of the differential inclusions in [7], [10] and [1], Chapter 5, Section 6 , where $\Pi_{K}$ is replaced by the set-valued mapping $x \mapsto-F(x)-N_{K}(x)$ from $K$ to the subsets of $X$. The first formulation of equation (1), in the form given above, appeared in [8] with $X:=\mathbb{R}^{n}$ and $K$ a convex polyhedral set. Is has been used, in the same finite dimensional context, in [9], [18], [19], [20], [21], [22], [26] just to cite a few. It was first formulated in infinite dimensional Hilbert spaces in [14] and [4].

Let $A C([0, \infty), K)$ denote the class of absolutely continuous functions defined on $[0, \infty)$ with values in $K$, which satisfy (1) for almost all $t \in[0, \infty)$. Cojocaru (see [4], Chapter 6, Theorem 6.1, and also Cojocaru and Jonker [5]) completely solved the problem of existence of solutions to 
a projected equation (1) on a Hilbert space of arbitrary dimension, in the class $A C([0, \infty), K)$. For completeness, we give the statement of the result.

Theorem 2.1. Let $X$ be a Hilbert space of arbitrary dimension and $K \subset X$ be a non-empty, closed and convex subset. Let $F: K \rightarrow X$ be a Lipschitz continuous vector field and $x_{0} \in K$. Then the initial value problem

$$
\frac{d x(t)}{d t}=\Pi_{K}\left(x(t),-F(x(t)), x(0)=x_{0} \in K\right.
$$

has a unique solution in $A C([0, \infty), K)$.

Definition 2.2. A projected dynamical system (PDS) is given by a mapping $\Phi: R_{+} \times K \rightarrow K$ which solves the initial value problem:

$$
\dot{\Phi}(t, x)=\Pi_{K}(\Phi(t, x),-F(\Phi(t, x))), \quad \Phi(0, x)=x \in K .
$$

We usually denote by $x(t):=\Phi(t, x)$ the solution of (2) starting at the initial point $x \in K$. There is a fundamental relation between a PrDE/PDS and a variational inequality problem. Let us call a point $x^{*} \in K$ with $\Pi_{K}\left(x^{*}, F\left(x^{*}\right)\right)=0$ a critical point for (1).

Theorem 2.2. Let $X$ be a Hilbert space of arbitrary dimension and $K \subset X$ be a non-empty, closed and convex subset. Let $F: K \rightarrow X$ be a vector field. Consider the variational inequality problem:

$$
\text { find } x \in K \text { such that }\langle F(x), y-x\rangle \geq 0, \forall y \in K \text {. }
$$

Then the solutions to the variational inequality problem coincide with the critical points of (1).

Proof. The proof can be found in [5], Theorem 2.2.

We note here that the relation between PDS and VI justifies the convention of using $-F$ in the definition of a PrDE/PDS, whereas $F$ is used to define the associated VI.

\section{The 1-dimensional obstacle problem as a variational inequality}

We present now an example of a variational inequality based on a problem of Physics. The problem is known in the literature (see [2], [16] for a more detailed description) as the obstacle problem. In this paper we only concern ourselves with the 1-dimensional case. 
3.1. The space $\boldsymbol{H}_{\mathbf{0}}^{\mathbf{1}}((\mathbf{0}, \boldsymbol{r}))$. Let $[0, r] \in \mathbb{R}$. We define the following sets of functions

$$
H^{1}((0, r)):=\left\{v \in A C((0, r), \mathbb{R}) \mid v^{\prime} \in L^{2}((0, r))\right\}
$$

and

$$
H_{0}^{1}((0, r)):=\left\{v \in H^{1}((0, r)) \mid v(0)=v(r)=0\right\},
$$

where $L^{2}((0, r))$ is the Hilbert space of measurable, $\|\cdot\|_{2}$-integrable functions on the set $(0, r)$.

The set $H_{0}^{1}((0, r))$ is a Sobolev Hilbert space (see for example [2], Chapter 5, Section 5.3, Theorem 5.12). An equivalent definition can be found in [16], Chapter 4, Definition 4.3, i.e., $H_{0}^{1}((0, r))$ is the closure of the set $C_{0}^{\infty}((0, r))$, of infinite differentiable functions with compact support in $(0, r)$, in the norm $\|v\|:=\|v\|_{L^{2}((0, r))}+\left\|v^{\prime}\right\|_{L^{2}((0, r))}$.

Let $a: H_{0}^{1}((0, r)) \times H_{0}^{1}((0, r)) \rightarrow R$ be the symmetric bilinear form given by $a(u, v)=\int_{0}^{r} u^{\prime}(x) v^{\prime}(x) d x$. In fact, $a$ is the inner product of the Hilbert space $H_{0}^{1}((0, r))$ (see [2], Chapter 5, Section 5.3, Theorem 5.13). The dual space of $H_{0}^{1}((0, r))$ is denoted by $H^{-1}((0, r))$ and is isomorphic to $H_{0}^{1}((0, r))$. The elements of $H^{-1}((0, r))$ are characterized as derivatives of functions in $L^{2}((0, r))$ in the distributional sense (see [16], Chapter 4, Section 4), namely for each $f \in H^{-1}((0, r))$, there exist $f_{0}, f_{1} \in L^{2}((0, r))$ so that for each $v \in H_{0}^{1}((0, r))$ we have $\langle f(u), v\rangle=\int_{0}^{r}\left\{f_{0} v-f_{1} v^{\prime}\right\} d x$. We denote by $\langle\cdot, \cdot\rangle: H^{-1}((0, r)) \times H_{0}^{1}((0, r)) \rightarrow \mathbb{R}$ the pairing between $H_{0}^{1}((0, r))$ and $H^{-1}((0, r))$, according to [16], Chapter 2. The bilinear form a defines a linear, continuous transformation $F: H_{0}^{1}((0, r)) \longrightarrow$ $H^{-1}((0, r))$, given by

$$
\langle F(u), v\rangle=a(u, v)=\int_{0}^{r} u^{\prime}(x) v^{\prime}(x) d x
$$

for any $u, v \in H_{0}^{1}((0, r))$. Is is immediate to see that $F$ is Lipschitz, since it is linear and there exists $c>0$ so that $\|F(u)\|_{H^{-1}((0, r))} \leq c\|u\|_{H_{0}^{1}((0, r))}$ ([2], Chapter 3, 26-27).

3.2. The obstacle problem: from optimization to VI. Consider a body $A \subset \mathbb{R}^{2}$, the obstacle, and two points $P_{1}$ and $P_{2}$ not belonging to $A$, as can be seen in Figure 1. We connect $P_{1}$ and $P_{2}$ by an elastic, weightless, homogeneous string that cannot penetrate $A$. The obstacle problem consists of finding the shape assumed by the string. 


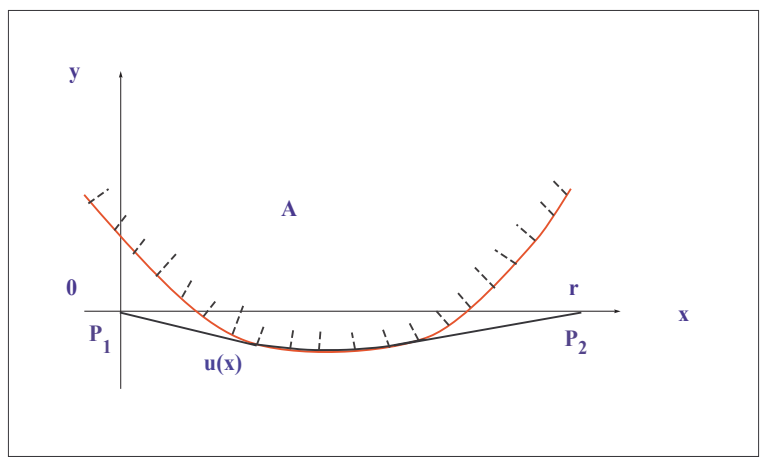

Figure 1

Suppose we introduce a system of cartesian axes with respect to which $P_{1}$ and $P_{2}$ have the coordinates $(0,0)$ and respectively, $(r, 0)$ and suppose that the lower part of the boundary of $A$, in the region we are interested (i.e. the interval $[0, r])$ is described by a curve of equation $y=\Psi(x)$. Let $y=u(x)$ be the curve describing the shape assumed by the string. Physics tells us that $u$ must be continuous, otherwise there would be a break in the string, but not necessarily differentiable, as shown in Figure 2.

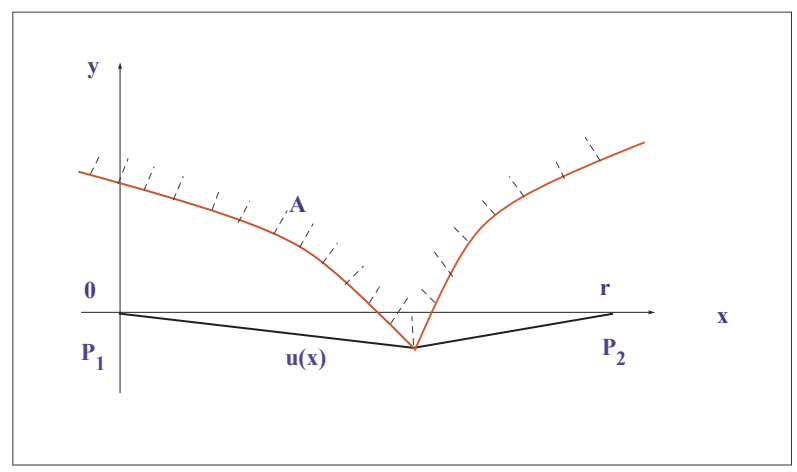

Figure 2

On the other hand, let us assume for now that $\Psi$ is continuous on $[0, r]$. Note that the obstacle problem can be written as an optimization problem because the string assumes the configuration which minimizes the energy of elastic deformation of the system, among all the configurations which are consistent with the constraints imposed on the string. The energy of deformation is defined as $e(v):=\frac{1}{2} \int_{0}^{r}\left[v^{\prime}(x)\right]^{2} d x$ ([2], Chapter 6 , 
Section 6.1). Then we can formulate the obstacle problem as

find $u \in K$ such that $e(u) \leq e(v)$, for all $v \in K$,

where $K:=\left\{v \in H_{0}^{1}((0, r)) \mid v \leq \Psi\right\}$ is the closed and convex set of possible configurations. To avoid $K$ being empty (see for example Figure 3), we impose further restrictions on $\Psi$, namely either:

1. $\Psi \in C^{0}((0, r)), \Psi\left(0^{+}\right)>0$ and $\Psi\left(r^{-}\right)>0$, or

2. $\Psi \in H^{1}((0, r)), \Psi(0) \geq 0$ and $\Psi(r) \geq 0$.

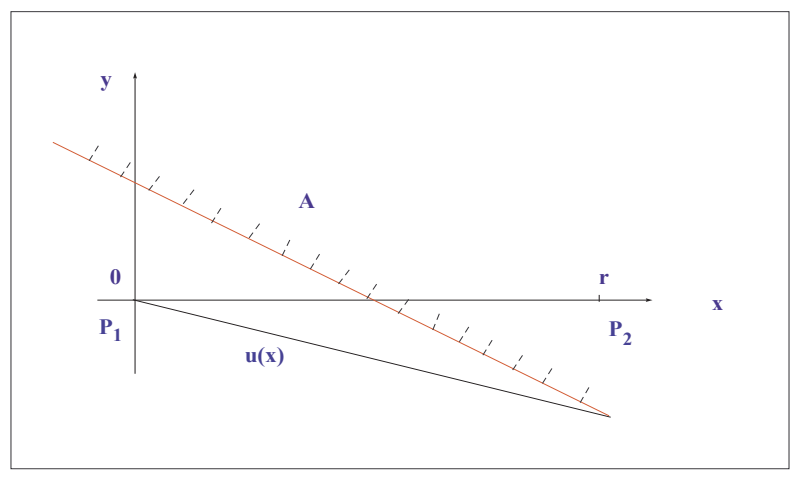

Figure 3

Now the formulation of the obstacle problem in the form of an optimization problem can be completed:

Problem 1. Suppose $\Psi$ satisfies either one of the conditions 1 or 2 above. Then the obstacle problem is that of

$$
\text { finding } u \in K \text { such that } e(u) \leq e(v) \text {, for all } v \in K \text {. }
$$

We consider again the mapping $F: H_{0}^{1}((0, r)) \rightarrow H^{-1}((0, r))$ given by $(3)$. Then the following result holds.

Lemma 3.1. Problem 1 is equivalent to the following variational inequality

$$
\text { find } u \in K \text { such that }\langle F(u), v-u\rangle \geq 0, \text { for all } v \in K .
$$

This VI problem has a unique solution, hence Problem 1 has a unique solution.

Proof. It is obvious that $e(v):=\frac{1}{2} a(v, v)$. Keeping in mind that $a$ is symmetric, the proof is a consequence of [2], Chapter 2, Section 2, Theorem 
2.3 , for $L:=0$. The symmetric bilinear form $a$ is coercive on $K$ (i.e., there exists $\alpha>0$ s.t. $a(u, u) \geq \alpha\|u\|^{2}$ ) and so the variational problem (4) has a unique solution, according to the Lions-Stampacchia Theorem (see [2], Chapter 3, Theorem 3.1).

\section{The 1-dimensional obstacle problem and PDS}

Variational inequalities theory offers solutions to static problems, like the one formulated in the previous section, also known as equilibrium solutions. If the problem is dynamic, the VI theory alone is not very helpful.

In our case, we suppose that the string is pinched, thus subjected to vibrations. The result of this experiment is that in a certain amount of time, in the absence of other actions, the string will regain its equilibrium position, i.e., that of minimal energy of deformation. We show here how an infinite-dimensional projected dynamics describes this experiment.

As in Section 2, we define the projected dynamical system associated to the 1-dimensional obstacle problem (4) in the VI form, given by the equation:

$$
\frac{d u(x, t)}{d t}=\Pi_{K}(u(x, t),-F(u(x, t))), u(x, 0)=u_{0}(x) \in K,
$$

where $F$ is defined as in (3). From Theorem 2.2, the critical points of (5) are the solutions to the VI (4), and by Lemma 3.1 there is only one such point for the PDS (5).

To analyze the movement of the string, in the presence of a unique equilibrium state, we use the theoretical results for PDS. We start by recalling the notions of monotonicity and pseudo-monotonicity, which are widely used in the theory of variational inequalities and complementarity, and in those of discontinuous differential equations and functional equations (see for example [1], [3], [4], [5], [6], [11], [12], [14], [16], [17], [18], [19]).

Definition 4.3. (1) Let $X$ be a Hilbert space, $\langle\cdot, \cdot\rangle$ the inner product on $X$ and $S \subset X$ a nonempty subset. The mapping $f: S \rightarrow X$ is called monotone on $S$ if, for any $x, y \in S$, we have $\langle f(x)-f(y), x-y\rangle \geq 0$.

(2) The mapping $f: S \rightarrow X$ is called pseudo-monotone on $S$ if, for any $x, y \in S$, we have $\langle f(y), x-y\rangle \geq 0 \Longrightarrow\langle f(x), x-y\rangle \geq 0$.

(3) Finally, $f$ is strongly pseudo-monotone on $S$ if, for any $x, y \in S$ there exists $\eta>0$ so that $\langle f(y), x-y\rangle \geq 0 \Longrightarrow\langle f(x), x-y\rangle \geq \eta\|x-y\|^{2}$.

Theorem 4.3. The mapping $F: H_{0}^{1}((0, r)) \rightarrow H^{-1}((0, r))$, given by $\langle F(u), v\rangle=a(u, v)$, for any $u, v \in H_{0}^{1}((0, r))$ is strongly pseudo-monotone on $K$. 
Proof. The strong pseudo-monotonicity of the mapping $F$ is given by the existence of a constant $\eta>0$ with the property that

$$
\langle F(u), v-u\rangle \geq 0 \Longrightarrow\langle F(v), v-u\rangle \geq \eta\|v-u\|^{2},
$$

or equivalently

$$
a(u, v-u) \geq 0 \Longrightarrow a(v, v-u) \geq \eta a(v-u, v-u),
$$

for any $u, v \in H^{1}((0, r))$. The second condition above can be re-written in terms of the energy of deformation as

$a(u, v)-2 e(u) \geq 0 \Longrightarrow 2 e(v)-a(v, u) \geq 2 \eta e(v-u)$, for any $u, v \in H^{1}((0, r))$.

We show next that $F$ is strongly pseudo-monotone with constant $\eta:=1$. By a simple calculation, keeping in mind the symmetry of the form $a$, we obtain

$$
\begin{array}{r}
2 e(u-v)=2 e(u)-a(u, v)+2 e(v)-a(v, u) \\
\Longrightarrow \quad 2 e(v)-a(v, u)-2 e(u-v)=a(u, v)-2 e(u) .
\end{array}
$$

Now evidently if $a(u, v)-2 e(u) \geq 0$, then $2 e(v)-a(u, v)-2 e(u-v) \geq 0$. Letting $\eta:=1$ we obtain that

$$
\langle F(v), u-v\rangle \geq 0 \Longrightarrow\langle F(u), u-v\rangle \geq\|u-v\|^{2},
$$

in other words $F$ is a strongly pseudo-monotone mapping on $K$.

In [18] for finite dimensions, and [14], [4] in infinite dimensions, the notions of (local or global) monotone attractors and strict monotone attractors were introduced to study the behaviour of a PDS around its steady states. These notions are different than the one of attractor, as used in the classical theory of dynamical systems, and are implied by various types of local or global monotonicity of the vector field $F$. In particular, the strict monotone attractors are especially important to study the existence of periodic cycles of a PDS (see [3] for strict monotone fields $F$ ), as Theorem 4.2 below shows.

In this paper, given Theorem 4.1, the field $F$ is globally strongly pseudomonotone, thus we remind only the definition of a global strict monotone attractor.

Definition 4.4. A critical point $u^{*}(\cdot)$ of $(5)$ is called a global strict monotone attractor if the mapping $t \mapsto\left\|u(\cdot, t)-u^{*}(\cdot)\right\|$ is decreasing, for any $u(\cdot, t)$ solution of $(5)$. 
Theorem 4.4. The following hold:

(1) The unique equilibrium $u^{*}$ of (5) is a strict monotone attractor which implies that the PDS (5) has no periodic cycles.

(2) The unique equilibrium $u^{*}$ of (5) is exponentially stable.

Proof. (1) From Theorem 4.1 together with [4], Theorem 7.2, or [14], Theorem 22, we have that the mapping $t \mapsto\left\|u(\cdot, t)-u^{*}(\cdot)\right\|$ is decreasing whenever $F$ is strongly, or merely strictly, pseudo-monotone. Let us assume that there exists at least one periodic cycle of (5), of period $T$, starting at a point $u(\cdot, 0) \in K$. Then we obtain that for each $t>0$,

$$
\left\|u(\cdot, t)-u^{*}(\cdot)\right\|=\left\|u(\cdot, t+T)-u^{*}(\cdot)\right\| .
$$

Since $t<t+T$, the above equality contradicts the fact that $t \mapsto$ $\left\|u(\cdot, t)-u^{*}(\cdot)\right\|$ is decreasing.

(2) Based on Theorem 4.1 and on [4], Theorem 7.6, the unique equilibrium of (5) is exponentially stable.

The interpretation of Theorem 4.2 is as follows. From Lemma 3.1, the VI problem (4) has a solution. Applying integration by parts we obtain that (4) is equivalent to the problem of finding $u \in H_{0}^{1}((0, r))$ such that $F(u)=-u^{\prime \prime}$ in the sense of distributions (see also [2], Chapter 6, Section 6.1). Hence the vector field $-F$ has the value $u^{\prime \prime}$ at any given function $u \in K$. Then for small deviations from a straight line, $u^{\prime \prime}$ is proportional to the curvature of the elastic string, and therefore to the vertical component of the tension on the elastic string towards its equilibrium position. Our conclusion here is that the motion of the string cannot be periodic in time, and that it converges to its rest position exponentially, the convergence taking place in the Sobolev space of functions $H_{0}^{1}((0, r))$.

\section{Concluding remarks and Acknowledgments}

The dynamic problem chosen to discuss here is experimentally simple, thus easy to grasp intuitively. However we see that the mathematics employed to describe it is quite rich and intertwines dynamical systems and nonlinear analysis. It is our belief that the methodology behind this example, that of associating an infinite-dimensional PDS to a VI, can be extremely useful for studying perturbations of steady states for a number of infinite-dimensional equilibrium problems.

The author would like to acknowledge here the visionary ideas of G. Isac as the catalyst for starting the rigorous infinite-dimensional PDS theory. This theory, together with its applications, prove to be rapidly expanding areas today. The author also acknowledges the useful suggestions of the referee. 


\section{References}

[1] J. P. Aubin and A. Cellina, Differential Inclusions, Springer-Verlag, Berlin, 1984.

[2] C. Baiocchi and A. Capelo, Variational and Quasivariational Inequalities: Applications to Free Boundary Problems, John Wiley and Sons, 1984.

[3] M. G. Cojocaru, Monotonicity and existence of periodic orbits for projected dynamical systems on Hilbert spaces, Proc. Amer. Math. Soc., 2005, to appear.

[4] M. G. Cojocaru, Projected dnamical Systems on Hilbert Spaces, Ph.D. Thesis, Queen's University, 2002.

[5] M. G. Cojocaru and L. B. Jonker, Projected differential equations in Hilbert spaces, Proc. Amer. Math. Soc., 132 (1) (2004), 183-193.

[6] M. G. Cojocaru, P. Daniele and A. Nagurney, Projected dynamical systems and evolutionary (Time-dependent) variational inequalities via Hilbert spaces with applications, J. Optim. Theory Appl., 2005, to appear.

[7] B. Cornet, Existence of slow solutions for a class of differential inclusions, J. Math. Anal. Appl., 96 (1983), 130-147.

[8] P. Dupuis and H. Ishii, On Lipschitz continuity of the solution mapping to the Skorokhod problem, with applications, Stochastics and Stochastics Reports, 35 (1990), 31-62.

[9] P. Dupuis and A. Nagurney, Dynamical systems and variational inequalities, Ann. Oper. Res., 44, (1993), 9-42.

[10] C. Henry, An existence theorem for a class of differential equations with multivalued righthand sides, J. Math. Anal. Appl., 41 (1973), 179-186.

[11] G. Isac, Topological Methods in Complementarity Theory, Kluwer Academic Publishers, 2000.

[12] G. Isac, Exceptional family of elements, feasibility and complementarity, J. Opt. Theory Appl., 104 (3) (2000), 577-588.

[13] G. Isac and M.G. Cojocaru, Functions without exceptional family of elements and the solvability of variational inequalities on unbounded sets, Topol. Methods Nonlin. Anal., 20 (2002), 375-391.

[14] G. Isac and M.G. Cojocaru, Variational inequalities, complementarity problems and pseudo-monotonicity: dynamical aspects, in Seminar on Fixed Point Theory Cluj-Napoca, Proceedings of the International Conference on Nonlinear Operators, Differential Equations and Applications, Babes-Bolyai University of Cluj-Napoca, III (2002), 4162. 
[15] G. Isac and M.G. Cojocaru, The projection operator in a hilbert space and its directional derivative, consequences for the theory of projected dynamical systems, J. Func. Spaces Appl., 2 (1) (2004), 71-95.

[16] D. Kinderlehrer and G. Stampacchia, An Introduction to Variational Inequalities and Their Applications, Academic Press, 1980.

[17] M.A. Krasnoselskii and P. P. Zabreiko, Geometrical Methods of Nonlinear Analysis, Springer-Verlag, A Series of Comprehensive Studies in Mathematics, 263 (1984).

[18] A. Nagurney and D. Zhang, On the stability of projected dynamical systems, J. Optim. Theory Appl., 85 (1995), 97-124.

[19] A. Nagurney and D. Zhang, Projected Dynamical Systems and Variational Inequalities with Applications, Kluwer Academic Publishers, 1996.

[20] A. Nagurney and S. Siokos, Financial Networks: Statics and Dynamics, Springer-Verlag, 1997.

[21] A. Nagurney, P. Dupuis and D. Zhang, A dynamical systems approach for network oligopolies and variational inequalities, Ann. Regional Science, 28 (1994), 263-283.

[22] A. Nagurney, T. Takayama and D. Zhang, Projected dynamical systems modelling and computation of spatial networks equilibria, Networks, 26 (1995), 69-85.

[23] A.S. Shapiro, Existence and differentiability of metric projections in Hilbert spaces, SIAM J. Optim., 4 (1994), 130-141.

[24] G. Stampacchia, Variational Inequalities, Theory and Applications of Monotone Operators, Proc. NATO Advanced Study Institute, Venice 1968, Ed. Oderisi, Gubbio, Italy, 1969, 101-192.

[25] E. Zarantonello, Projections on convex sets in Hilbert space and spectral theory, Contributions to Nonlinear Functional Analysis, Publ. No. 27, Math. Res. Center, Univ. Wisconsin, Academic Press, 1971, 237-424.

[26] D. Zhang and A. Nagurney, Formulation, stability and computation of traffic network equilibria as projected dynamical systems, J. Opt. Theory Appl., 93 (2) (1997), 417-444.

Department of Mathematics and Statistics

MacNaughton Hall, Room 548

University of Guelph

Guelph, Ontario

Canada

(E-mail : mcojocar@uoguelph.ca) 


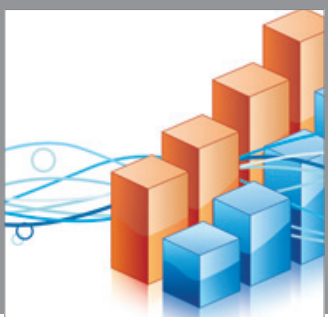

Advances in

Operations Research

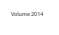

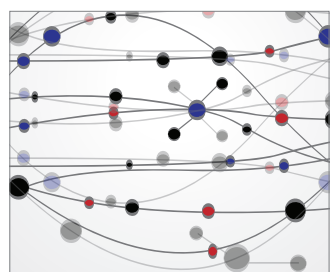

\section{The Scientific} World Journal
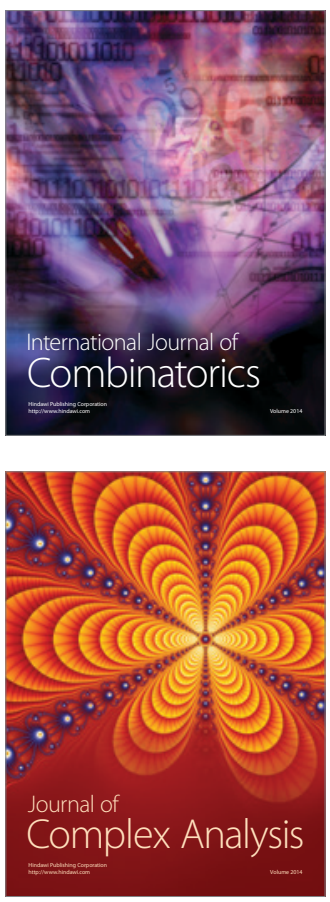

International Journal of

Mathematics and

Mathematical

Sciences
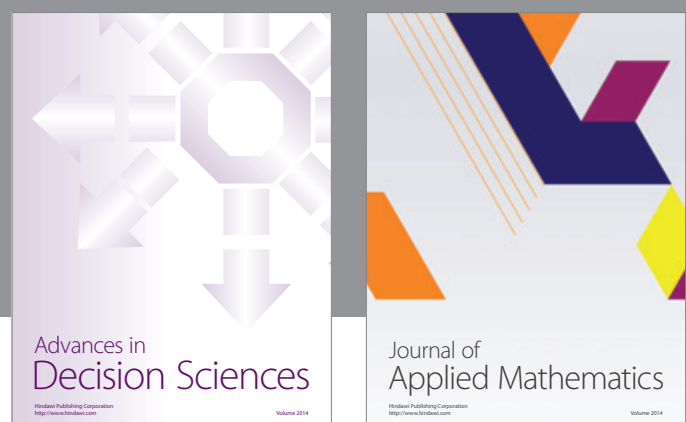

Journal of

Applied Mathematics
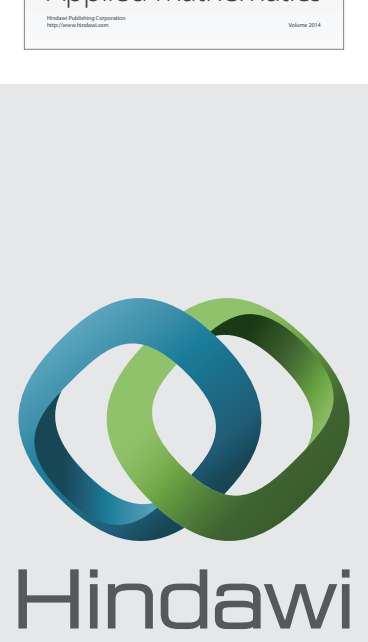

Submit your manuscripts at http://www.hindawi.com
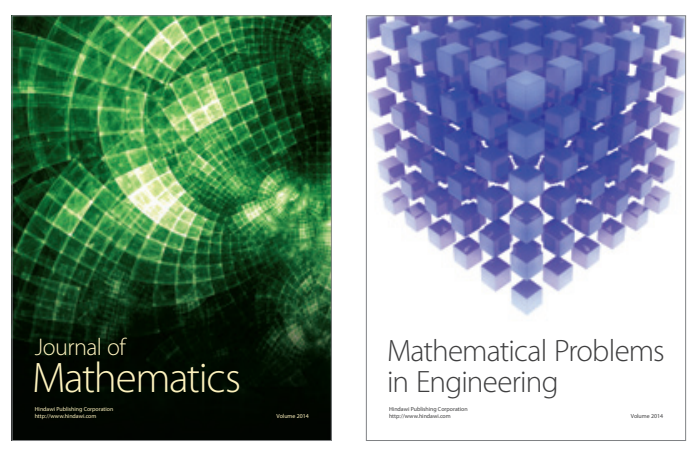

Mathematical Problems in Engineering
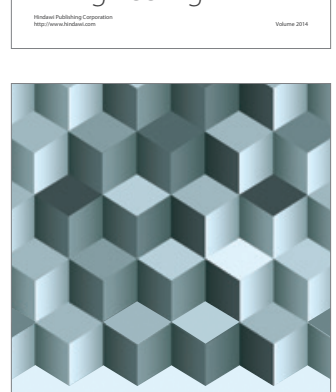

Journal of

Function Spaces
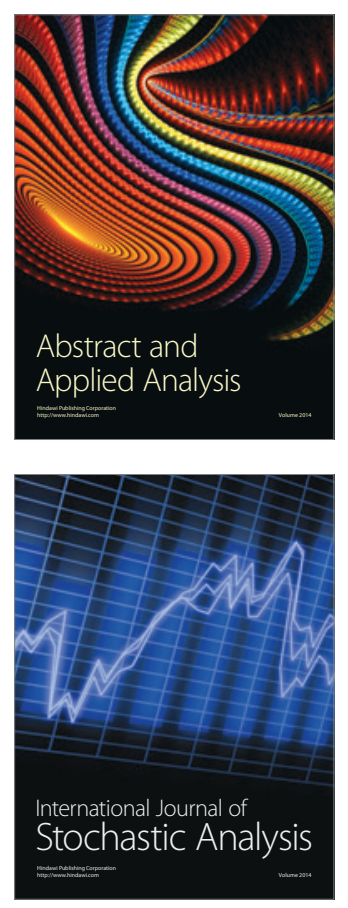

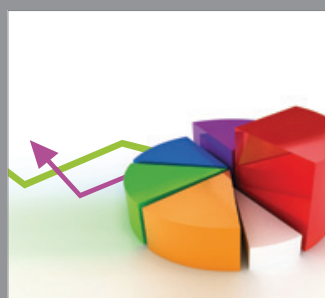

ournal of

Probability and Statistics

Promensencen
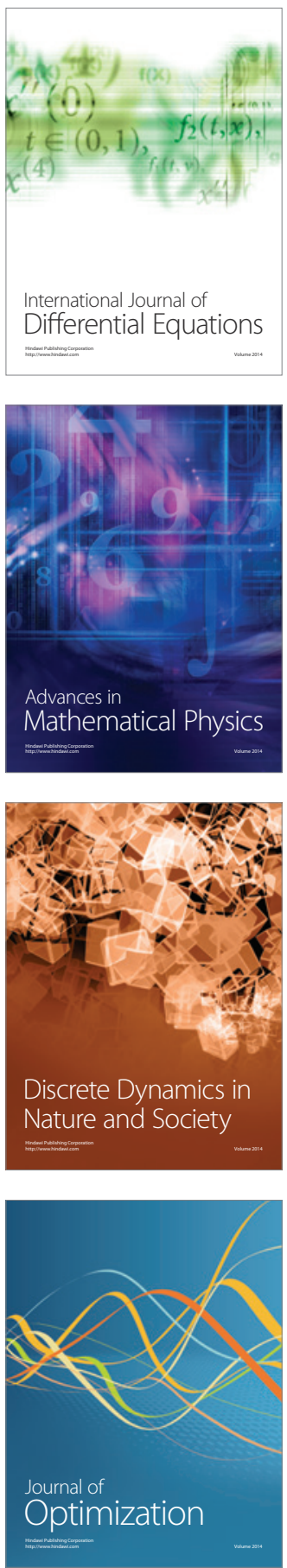